$11-1-2016$

\title{
Comparison of Some Multivariate Nonparametric Tests in Profile Analysis to Repeated Measurements
}

Mehrdad Vossoughi

Shiraz University of Medical Sciences, Shiraz, Iran, vossoughim@sums.ac.ir

Shila Shahvali

Fars Planning and Budget Organization, Shiraz, Iran

Erfan Sadeghi

Isfahan University of Medical Sciences, Isfahan, Iran

Follow this and additional works at: http://digitalcommons.wayne.edu/jmasm

Part of the Applied Statistics Commons, Social and Behavioral Sciences Commons, and the Statistical Theory Commons

\section{Recommended Citation}

Vossoughi, Mehrdad; Shahvali, Shila; and Sadeghi, Erfan (2016) "Comparison of Some Multivariate Nonparametric Tests in Profile Analysis to Repeated Measurements," Journal of Modern Applied Statistical Methods: Vol. 15 : Iss. 2 , Article 19.

DOI: $10.22237 /$ jmasm/1478002620

Available at: http://digitalcommons.wayne.edu/jmasm/vol15/iss2/19

This Regular Article is brought to you for free and open access by the Open Access Journals at DigitalCommons@WayneState. It has been accepted for inclusion in Journal of Modern Applied Statistical Methods by an authorized editor of DigitalCommons@WayneState. 


\section{Comparison of Some Multivariate Nonparametric Tests in Profile Analysis to Repeated Measurements}

\section{Cover Page Footnote}

Acknowledgements: The authors thank Dr. Shahram Hamedani of the Center for Research Improvement of the School of Dentistry for improving the use of English in the manuscript. 


\section{Comparison of Some Multivariate Nonparametric Tests in Profile Analysis to Repeated Measurements}

\author{
Mehrdad Vossoughi \\ Shiraz University of Medical \\ Sciences \\ Shiraz, Iran
}

Shila Shahvali

Bureau of Statistics and Information, Fars

Planning and Budget Organization

Shiraz, Iran

\author{
Erfan Sadeghi \\ Isfahan University of Medical \\ Sciences \\ Isfahan, Iran
}

Through Monte Carlo simulations, the performance of six multivariate nonparametric tests for testing the hypothesis of parallelism in profile analysis was studied. In conclusion, the tests based on ranks were as efficient as Hotelling's $T^{2}$ under multivariate normal distribution. For the heavy tailed distribution, the tests based on signs performed best.

Keywords: $\quad$ Monte Carlo simulation, multivariate, nonparametric, profile analysis, heavy tailed

\section{Introduction}

Research in many areas of application frequently involves repeated measurements in which response from each experimental unit is measured repeatedly over different occasions such as time points. The linear mixed model to repeated measurements (Laird \& Ware, 1982; Ware, 1985) was developed to analyze incomplete and unbalanced data. However, the performance of this complex approach is highly sensitive to the choice of model for mean function and correlation structure for errors (Littell, Pendergast, \& Natarajan, 2000; Park, Park, \& Davis, 2001; Vossoughi, Ayatollahi, Towhidi, \& Ketabchi, 2012). Although several nonparametric methods have been developed for non-normal responses (Azzalini \& Bowman, 1991; Singer, Poleto, \& Rosa, 2004; Wernecke \& Kalb, 1999; Wernecke \& Kaufmann, 2000), model building and software implementation of these methods are extremely complicated.

Due to these difficulties, investigators are often interested in using the traditional approaches especially when the circumstances are controlled for

Dr. Vossoughi is an Assistant Professor in the Department of Dental Public Health.

Email himat:vossoughim@sums.ac.ir. 


\section{MULTIVARIATE NONPARAMETRIC TESTS IN PROFILE ANALYSIS}

obtaining complete data. In this context, the profile analysis method using MANOVA tests makes no assumption regarding the correlation structure and trend of mean model and hence is widely used. Nevertheless, the MANOVA tests perform poorly when the distribution of errors much deviates from multivariate normal (Davis, 1980, 1982; Everitt, 1979; Olson, 1974; Um \& Randles, 1998).

Bhapkar (1984) and Sen (1984) discussed asymptotically distribution-free analogous of profile analysis. Multivariate extensions of Kruskal-Wallis and Brown-Mood median tests based on marginal ranks and signs were discussed in Puri and Sen (1971) but suffer from a lack of invariance with respect to affine transformations. Several authors provided detailed descriptions of affine invariant and non-invariant competitors based on spatial signs and ranks (Hettmansperger, Möttönen \& Oja, 1998; Hettmansperger \& Oja, 1994; Möttönen \& Oja, 1995; Oja, 1999; Oja \& Randles, 2004). The asymptotic efficiency of multivariate spatial sign and rank tests were studied by Möttönen, Oja, and Tienari (1997), Möttönen, Hettmansperger, Oja, and Tienari (1998), Nordhausen, Oja, and Tyler (2006) and Oja and Randles (2004). The theory and software implementation of affine invariant/non-invariant spatial sign and rank tests were well described by Oja (2010).

The aim of this study is to compare the performance of six nonparametric multivariate multi-sample tests with Hotelling's $T^{2}$ in profile analysis for repeated measurements. For this propose, Monte Carlo simulations based on broad spectrum of scenarios are used to study the empirical type I error rates and powers of the tests in testing the hypothesis of parallelism. Affine/non-affine invariant multivariate generalizations of multi-sample tests are compared based on spatial scores discussed in Oja (2010, Ch. 11) and multivariate generalization of multisample tests based on marginal scores discussed in Chapter 5 of Puri and Sen (1971).

Although the test of group main effect or hypothesis that the two groups are at the same level can also be assessed using multivariate multi-sample procedures, it was not included in the simulations for three priori reasons. First, rather than testing the general multivariate hypothesis $\boldsymbol{\mu}_{1}=\boldsymbol{\mu}_{2}=\ldots=\boldsymbol{\mu}_{k}$ to assess group main effect, summarizing the response vector of each subjects using its individual mean and then applying univariate tests is generally implemented in a parametric profile analysis (Davis, 2002; Rencher, 1995). Second, the performance of Hotelling's $T^{2}$ and its nonparametric counterparts were studied to test above general hypothesis (Möttönen et al., 1998; Nordhausen et al., 2006; Um \& Randles, 1998). Finally, group main effect has no direct interpretation in the presence of significant interaction and hence is not the primary hypothesis of interest in profile analysis. 


\section{VOSSOUGHI ET AL.}

Although the Monte Carlo comparison of methods for the analysis of repeated measurements has been an active area of research (Bhapkar \& Patterson, 1978; Marcucci, 1986; Mendoza, Toothaker, \& Nicewander, 1974; Park et al., 2001; Schwertman, Flynn, Stein, \& Schenk, 1985; Schwertman, Fridshal, \& Magrey, 1981), this study has been designed to examine some different aspects. First, the performances of recent nonparametric tests based on spatial signs and ranks considered here have not yet been studied in the area of profile analysis. Second, the effect of various correlation structures for errors has not included by most of the previous literature on this subject. Finally, the performance of the non-invariant tests under various transformation matrices widely used in the profile analysis are examined.

\section{Methodology}

\section{Parametric profile analysis}

The structure of profile analysis for the analysis of repeated measurements is now considered. Suppose that repeated measurements have been taken from $k$ groups of subjects at $p$ occasions. Let $\mathbf{y}_{i j}=\left(y_{i j 1}, \ldots, y_{i j p}\right)^{\mathrm{T}}$ represent the response vector from the $j^{\text {th }}$ subject in group $i$ for $j=1, \ldots, n_{k}, i=1, \ldots, k$. The profile analysis model is

$$
\mathbf{y}_{i j}=\boldsymbol{\mu}_{i}+\varepsilon_{i j},
$$

where the vector $\boldsymbol{\varepsilon}_{i j}=\left(\varepsilon_{i j 1}, \ldots, \varepsilon_{i j p}\right)^{T}$ is the vector of errors for the $j^{\text {th }}$ subject in group $I$ and $\boldsymbol{\mu}_{i}=\left(\mu_{i 1}, \ldots, \mu_{i p}\right)^{T}$ is the population mean vector for the $i^{\text {th }}$ group. Error vectors are assumed to be independent and normally distributed with mean vector $\mathbf{0}$ and common covariance matrix $\boldsymbol{\Sigma}$.

Arguably, in the presence of group $\times$ occasion interaction, the tests of main effects are confounded. Therefore, the primary aim in the profile analysis is to test the hypothesis of parallelism of $k$ group profiles. The test of the hypothesis can be constructed as

$$
\mathrm{H}_{0}=\mathbf{C} \boldsymbol{\mu}_{1}=\ldots=\boldsymbol{C} \boldsymbol{\mu}_{k} \text { or } \boldsymbol{\mu}_{1}^{*}=\ldots=\boldsymbol{\mu}_{k}^{*} \text {, }
$$




\section{MULTIVARIATE NONPARAMETRIC TESTS IN PROFILE ANALYSIS}

where $\boldsymbol{\mu}_{h}^{*}$ is the mean of transformed observations, $\mathbf{y}_{i j}^{*}=\mathbf{C} \mathbf{y}_{i j}$. Here, $\mathbf{C}$ is a $p-1 \times p$ transformation matrix with rank $p-1$ satisfying $\mathbf{C}_{\mathbf{1}}=\mathbf{0}$, where $\mathbf{1}$ is the unit matrix. For instance, when $p=3$, three widely-used matrices are:

$\mathbf{C}_{1}$ : Mean difference $\quad \mathbf{C}_{2}$ : Adjacent difference $\quad \mathbf{C}_{3}$ : Last-value difference

$$
\frac{1}{3}\left[\begin{array}{ccc}
2 & -1 & -1 \\
-1 & 2 & -1
\end{array}\right] \quad\left[\begin{array}{ccc}
-1 & 1 & 0 \\
0 & -1 & 1
\end{array}\right] \quad\left[\begin{array}{ccc}
1 & 0 & -1 \\
0 & 1 & -1
\end{array}\right]
$$

For example, the analogous hypothesis of parallelism for $k=2$ and the transformation matrix $\mathbf{C}_{2}$ is

$$
H_{0}:\left(\begin{array}{c}
\mu_{12}-\mu_{11} \\
\mu_{13}-\mu_{12} \\
\vdots \\
\mu_{1 p}-\mu_{1, p-1}
\end{array}\right)=\left(\begin{array}{c}
\mu_{22}-\mu_{21} \\
\mu_{23}-\mu_{22} \\
\vdots \\
\mu_{2 p}-\mu_{2, p-1}
\end{array}\right) \text {. }
$$

Then, one-way multivariate analysis of variance (MANOVA) test statistics such as Wilk's $\Lambda$ (if $k>2$ ) or Hotelling's $T^{2}$ (if $k=2$ ) can be used to assess the equality of mean vectors of transformed variables $\mathbf{y}_{i j}^{*}$ or equivalently hypothesis of parallelism. Similarly, nonparametric multivariate tests can be applied on the transformed observations to assess the equality of population locations when the underlying distribution deviates from normality.

\section{Nonparametric counterparts of MANOVA tests}

A brief overview of six nonparametric multivariate multi-sample tests used for profile analysis in the Monte Carlo simulations are now considered. The focus is primarily on recent methods that are supplied in standard statistical software packages. Here, we assume the $p$-dimensional data vectors are generated independently using model

$$
\mathbf{y}_{i j}=\boldsymbol{\theta}_{i}+\boldsymbol{\varepsilon}_{i j},
$$

where $\boldsymbol{\theta}_{i}$ denotes the $p$-dimensional location vector for group $i$ which is not necessary the corresponding mean vector and $\boldsymbol{\varepsilon}_{i j}$ is the vector of errors from an 


\section{VOSSOUGHI ET AL.}

elliptical multivariate distribution with location vector $\mathbf{0}$ and scatter matrix $\boldsymbol{\Sigma}$. When measurements are not normally distributed, nonparametric multi-sample multivariate tests can be employed to test the hypothesis of no group $\times$ occasions interaction effect as

$$
H_{0}=\boldsymbol{\theta}_{1}^{*}=\ldots=\boldsymbol{\theta}_{k}^{*},
$$

where $\boldsymbol{\theta}_{i}^{*}$ indicates the location vector of transformed variables from group $i$.

\section{Tests based on spatial signs}

The test statistic based on spatial signs for testing $H_{0}$ is

$$
Q=\sum_{i=1}^{c}\left\{n_{i} \overline{\mathbf{U}}_{i}^{* \prime} \overline{\mathbf{U}}_{i}^{*}\right\}
$$

where $\bar{U}_{i}^{*}$ denotes the sample mean vector of spatial signs transformed using inner centering and outer standardization. Although the test is location invariant, it is not affine invariant; that is the condition $Q(\mathbf{A Y})=Q(\mathbf{Y})$ is not satisfied for every nonsingular matrix $\mathbf{A}$ with rank $p$.

The affine invariant test statistic is

$$
Q=p \sum_{i=1}^{c}\left\{n_{i} \overline{\mathbf{U}}_{i}^{*^{\prime}} \overline{\mathbf{U}}_{i}^{*}\right\}
$$

where, here, $\overline{\mathbf{U}}_{i}^{*}$ is the sample mean vector of spatial signs transformed using inner centering and inner standardization.

The test statistics are multivariate generalizations of two- and severalsample Mood's median test and are asymptotically distributed as $\chi_{(c-1) p}^{2}$ when $H_{0}$ is true. The spatial sign tests are denoted by SS and SSI for the non invariant and invariant versions in the simulations, respectively. See Oja (2010) regarding the theory and software implementation of spatial sign and rank tests.

\section{Tests based on spatial ranks}

The constructions of tests based on spatial ranks are essentially the same as the spatial sign cases, with the difference that $\overline{\mathbf{U}}_{i}^{*}$ 's are replaced by the corresponding 


\section{MULTIVARIATE NONPARAMETRIC TESTS IN PROFILE ANALYSIS}

sample mean vector of transformed spatial ranks, $\overline{\mathbf{R}}_{i}^{*}$. Due to the fact that the spatial ranks are naturally centered, one needs only to standardize them using outer or inner approaches to construct non affine or affine invariant versions of test statistic. The test statistics using outer and inner standardization are in the form of

$$
Q=\sum_{i=1}^{c}\left\{n_{i} \overline{\boldsymbol{R}}_{i}^{* \prime} \overline{\boldsymbol{R}}_{i}^{*}\right\}
$$

and

$$
Q=p \sum_{i=1}^{c}\left\{n_{i} \overline{\boldsymbol{R}}_{i}^{* \prime} \overline{\boldsymbol{R}}_{i}^{*}\right\}
$$

respectively. The asymptotic null distribution of both test statistics is $\chi_{(c-1) p}^{2}$. The non invariant and affine invariant spatial rank tests are denoted by $S R$ and $S R I$ in the simulations, respectively.

\section{Tests based on marginal ranks and signs}

The multivariate multi-sample rank sum test compares the difference between the sample average rank vector $\overline{\mathbf{r}}_{i}$ and the combined-data average rank vector $\overline{\mathbf{r}}$ as

$$
L_{R}=\sum_{i=1}^{c} n_{i}\left(\overline{\mathbf{r}}_{i}-\overline{\mathbf{r}}\right)^{\prime} \mathbf{V}^{-1}\left(\overline{\mathbf{r}}_{i}-\overline{\mathbf{r}}\right)
$$

The test reduces to the Kruskal-Wallis test when $p=1$ and to WilcoxonMann-Whitney test when $p=1$ and $c=2$.

The multivariate multi-sample median test uses the corresponding average vectors based on sample signs (computed regarding combined-data median vector) to test the null hypothesis as

$$
L_{S}=\sum_{i=1}^{c} n_{i}\left(\overline{\mathbf{s}}_{i}-\overline{\mathbf{s}}\right)^{\prime} \mathbf{V}^{-1}\left(\overline{\mathbf{s}}_{i}-\overline{\mathbf{s}}\right)
$$

Write $\mathbf{V}$ to denote the sample covariance matrix of marginal ranks and signs in $L_{R}$ and $L_{S}$, respectively. The asymptotic null distribution of both statistics is 


\section{VOSSOUGHIET AL.}

$\chi_{(c-1) p}^{2}$. The multivariate multi-sample location tests based on the vector of marginal signs and ranks were discussed in detail by Puri and Sen (1971).

The marginal sign and rank tests are denoted by $M S$ and $M R$, respectively, in the simulation.

\section{Simulation study}

The structure of a Monte Carlo study used to investigate the performances of tests according to empirical type I error rates and powers is now discussed. The profile model (4) with two groups $(k=2)$, number of measurements $p=4,8$ and sample sizes $n=10,20$ and 30 for each of the two samples was considered. The performances of MANOVA test (here Hotelling's $T^{2}$ since $k=2$ ) and the six nonparametric counterparts in testing the hypothesis of parallelism were compared under various scenarios. In the simulations, Hotelling's $T^{2}$ test was denoted by $T^{2}$.

Consider three types of correlation structures for errors; compound symmetry (CS) with $\rho=0.2$, first-order autoregressive (AR1) with $\rho=0.5$, and an unstructured model (UN). The UN structure considered here was an arbitrary $p \times p$ correlation matrix producing a positive definite covariance matrix. Errors were generated from multivariate $t$ with 3 degrees of freedom (denoted by $t$ (3)) as a heavy-tailed distribution and multivariate normal distribution with mean vector $\mathbf{0}$ and variances 3 for above correlation structures. Therefore the two distributions had the same mean vector and covariance matrix and differ only by degrees of heaviness of their tails. The MANOVA tests have been shown to have low powers when the underlying distribution is heavy-tailed, in particular (see e.g. (Somorčík, 2006). The reason is that the sample mean vector and covariance matrix would not provide proper estimates of location and variation under the presence of outliers (see, e.g. Um \& Randles, 1998).

Throughout the simulations, $\boldsymbol{\theta}_{1}$ was considered to be a zero vector. To compute the empirical type I error rates, data were simulated under the hypothesis of parallelism, $H_{0}: \mathbf{C} \boldsymbol{\theta}_{1}=\mathbf{C} \boldsymbol{\theta}_{2}$, when $\boldsymbol{\theta}_{2}$ was also considered to be a zero vector. However, the hypothesis of interaction or $H_{1}: \mathbf{C} \boldsymbol{\theta}_{1} \neq \mathbf{C} \boldsymbol{\theta}_{2}$ was simulated when $\boldsymbol{\theta}_{2}=(0,1,1,0)^{T}$ and $(0,0,1,1,1,1,0,0)^{T}$ for $p=4$ and 8 , respectively; so that the empirical powers were computed. Also considered are the three transformation matrices $\mathbf{C}_{1}$ to $\mathbf{C}_{3}$ presented above to evaluate the robustness of non affine-invariant tests.

For each combination of above scenarios, 1000 replications were carried out and significance level was considered to be 0.05 . All simulations performed using 


\section{MULTIVARIATE NONPARAMETRIC TESTS IN PROFILE ANALYSIS}

R 3.0.1 (R Development Core Team, 2013). In this respect, the multi-sample tests implemented using the R packages MNM, ICSNP, and Hotelling. Multivariate normal and $t$ data were generated using the R packages MASS and mvtnorm, respectively.

\section{Results}

Displayed in Tables 1 and 2 are empirical type I error rates of the tests for errors generated from multivariate normal and multivariate $t(3)$ distributions, respectively. Each value is the proportion of 1000 replications for which the hypothesis of parallelism or null hypothesis was incorrectly rejected. In general, all tests preserved the nominal 5 percent level under all scenarios. However, for $p=8$ and smaller sample size $n=10$, the type I error rates of nonparametric tests were smaller than those of parametric one.

Displayed in Table 3 are empirical powers of the test for multivariate normal distribution. Each power value computed as the proportion of 1000 replications for which the hypothesis of parallelism was correctly rejected. In summary, among the tests, the affine invariant and non-invariant tests based on spatial ranks as well as test based on marginal ranks reached a power level fully close to that of Hotelling $T^{2}$ in which the differences were considerably negligible for all correlation structures. However, for the smaller sample size $n=10$ and larger number of replication $p=8$, the amount of difference somewhat increased. The test based on marginal signs performed unsatisfactorily; that is its powers were much lower than those of other test statistics for all correlation structures and transformation matrices. Interestingly, for all transformation matrices, the competitor based on spatial signs dominated the test based on marginal sign and was comparable to the best tests in the multivariate normal case. The empirical power trends of tests for multivariate normal distribution are visualized in Figure 1.

Shown in Table 4 are empirical powers of the test for data generated from multivariate $t(3)$ as a heavy tailed distribution. The results showed that the tests based on spatial signs and ranks and tests based on marginal ranks fully dominated Hotelling's $T^{2}$ for larger sample sizes $n=20$ and 30 and any given correlation structure. For a fixed sample size, the amount of superiority somewhat decreased as $p$ increased. In summary, the tests based on spatial signs yielded the greater values than the counterparts based on spatial and marginal ranks. Note that for a fixed $p$, the larger the size of sample, the greater the amount of difference in power levels. However, the performance of marginal sign test 


\section{VOSSOUGHI ET AL.}

Table 1. The empirical type I error rates of tests under multivariate normal distribution.

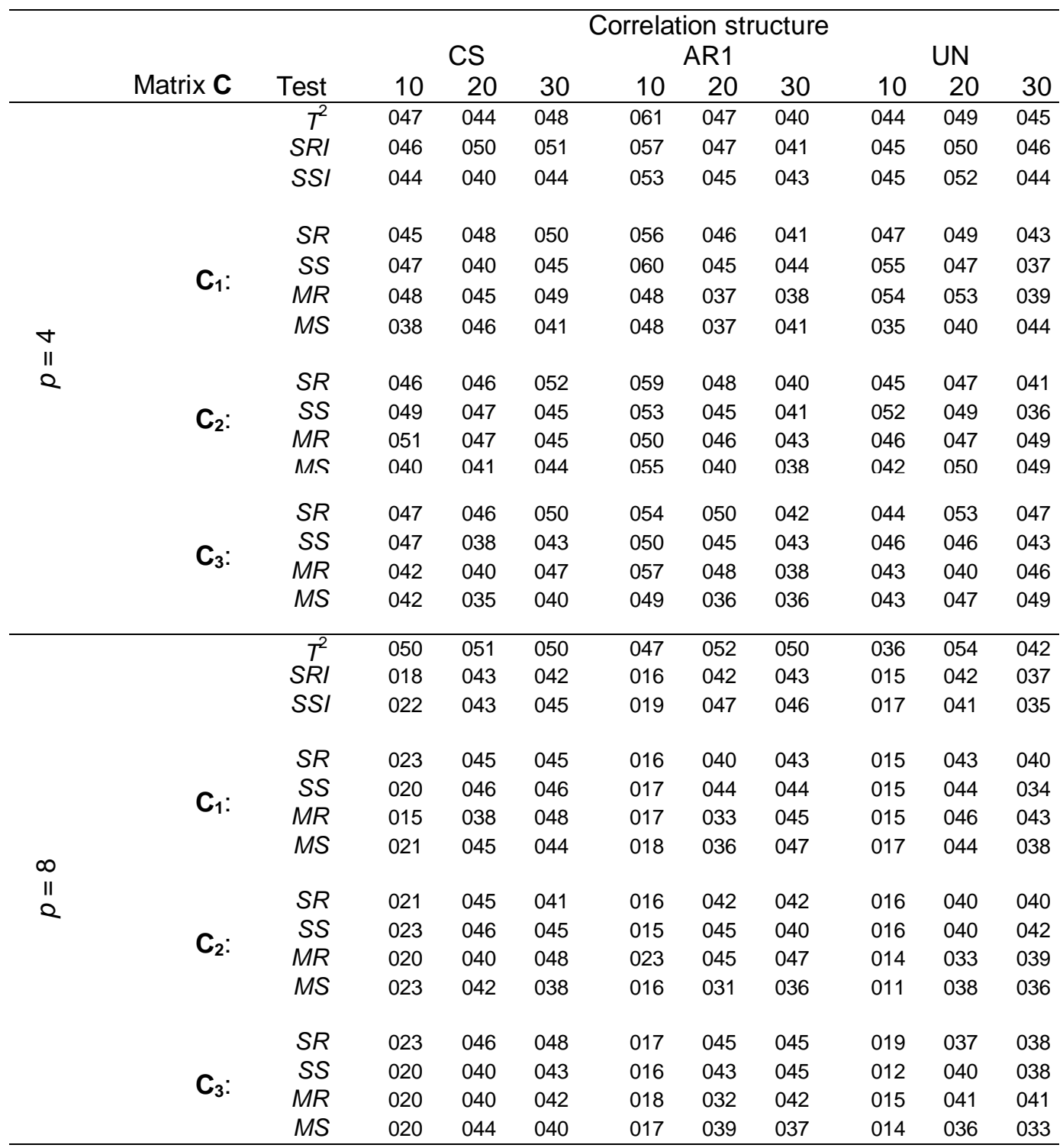

*Note: The entries within table correspond to empirical type I error rates multiplied by 1000.

was unsatisfactory since it was just as efficient as Hotelling $T^{2}$ for some specific choices of C. Surprisingly; even the permutation procedure provided no additional gain in efficiency for Hotelling's $T^{2}$ under the heavy tailed distribution and hence not reported here. 


\section{MULTIVARIATE NONPARAMETRIC TESTS IN PROFILE ANALYSIS}

Table 2. The empirical type I error rates of tests under multivariate $t$ distribution.

\begin{tabular}{|c|c|c|c|c|c|c|c|c|c|c|c|}
\hline & \multirow[b]{3}{*}{ Matrix C } & \multirow[b]{3}{*}{ Test } & \multicolumn{9}{|c|}{ Correlation structure } \\
\hline & & & \multicolumn{3}{|c|}{ CS } & \multicolumn{3}{|c|}{ AR1 } & \multicolumn{3}{|c|}{ UN } \\
\hline & & & 10 & 20 & 30 & 10 & 20 & 30 & 10 & 20 & 30 \\
\hline \multirow{16}{*}{$\begin{array}{l}\nabla \\
\text { II } \\
2\end{array}$} & \multirow{8}{*}{$\mathbf{C}_{1}:$} & $T^{2}$ & 040 & 036 & 043 & 034 & 045 & 047 & 047 & 046 & 038 \\
\hline & & $S R I$ & 051 & 049 & 050 & 048 & 056 & 047 & 057 & 054 & 046 \\
\hline & & SSI & 047 & 054 & 051 & 044 & 055 & 047 & 046 & 051 & 061 \\
\hline & & $S R$ & 052 & 046 & 056 & 046 & 056 & 050 & 054 & 052 & 050 \\
\hline & & SS & 051 & 049 & 060 & 048 & 052 & 050 & 047 & 053 & 054 \\
\hline & & $M R$ & 046 & 043 & 049 & 047 & 046 & 050 & 048 & 054 & 041 \\
\hline & & MS & 050 & 049 & 049 & 046 & 057 & 057 & 046 & 051 & 039 \\
\hline & & & & & & & & & & & \\
\hline & \multirow{4}{*}{$\mathbf{C}_{2}$ : } & $S R$ & 048 & 048 & 051 & 047 & 055 & 048 & 053 & 053 & 051 \\
\hline & & $S S$ & 045 & 052 & 050 & 050 & 052 & 046 & 050 & 054 & 057 \\
\hline & & $M R$ & 048 & 048 & 055 & 043 & 047 & 050 & 052 & 056 & 052 \\
\hline & & $M S$ & 049 & 044 & 049 & 040 & 048 & 062 & 050 & 045 & 043 \\
\hline & \multirow{4}{*}{$\mathrm{C}_{3}$ : } & $S R$ & 046 & 050 & 046 & 048 & 057 & 050 & 054 & 055 & 050 \\
\hline & & SS & 048 & 058 & 044 & 053 & 051 & 042 & 044 & 054 & 062 \\
\hline & & $M R$ & 042 & 055 & 051 & 042 & 047 & 047 & 050 & 055 & 055 \\
\hline & & $M S$ & 043 & 047 & 046 & 049 & 049 & 041 & 047 & 055 & 059 \\
\hline \multirow{16}{*}{$\begin{array}{l}\infty \\
\| \\
2\end{array}$} & \multirow{7}{*}{$\mathbf{C}_{1}:$} & $T^{2}$ & 044 & 033 & 033 & 037 & 034 & 051 & 034 & 028 & 044 \\
\hline & & $S R I$ & 022 & 036 & 029 & 013 & 031 & 051 & 021 & 031 & 041 \\
\hline & & SSI & 019 & 036 & 038 & 019 & 040 & 044 & 031 & 034 & 046 \\
\hline & & $S R$ & 019 & 034 & 027 & 010 & 030 & 049 & 020 & 030 & 045 \\
\hline & & SS & 015 & 031 & 039 & 019 & 043 & 041 & 018 & 035 & 049 \\
\hline & & $M R$ & 018 & 028 & 038 & 014 & 026 & 029 & 021 & 030 & 045 \\
\hline & & $M S$ & 020 & 038 & 038 & 015 & 035 & 038 & 013 & 029 & 036 \\
\hline & & & & & & & & & & & \\
\hline & \multirow{4}{*}{$\mathrm{C}_{2}$} & $S R$ & 018 & 035 & 027 & 011 & 032 & 053 & 019 & 029 & 042 \\
\hline & & SS & 017 & 039 & 038 & 017 & 041 & 046 & 025 & 032 & 041 \\
\hline & & $M R$ & 021 & 037 & 032 & 019 & 030 & 044 & 014 & 033 & 047 \\
\hline & & $M S$ & 015 & 037 & 039 & 015 & 034 & 045 & 018 & 031 & 049 \\
\hline & \multirow{4}{*}{$\mathrm{C}_{3}:$} & $S R$ & 020 & 027 & 026 & 012 & 033 & 046 & 019 & 028 & 042 \\
\hline & & SS & 014 & 030 & 040 & 012 & 039 & 044 & 020 & 033 & 047 \\
\hline & & $M R$ & 012 & 022 & 022 & 013 & 042 & 048 & 018 & 032 & 038 \\
\hline & & $M S$ & 020 & 037 & 038 & 012 & 038 & 043 & 018 & 026 & 044 \\
\hline
\end{tabular}

*Note: The entries within table correspond to empirical type I error rates multiplied by 1000.

Although not reported in the tables, additional simulations demonstrated that the superiority of nonparametric tests was not attained until $n$ reached 15 . Figure 2 shows the empirical power trends of tests for the heavy tailed distribution. 


\section{VOSSOUGHI ET AL.}

Table 3. The empirical powers of tests under multivariate normal distribution.

\begin{tabular}{|c|c|c|c|c|c|c|c|c|c|c|c|}
\hline & \multirow[b]{3}{*}{ Matrix $\mathbf{C}$} & \multirow[b]{3}{*}{ Test } & \multicolumn{9}{|c|}{ Correlation structure } \\
\hline & & & \multicolumn{3}{|c|}{ CS } & \multicolumn{3}{|c|}{ AR1 } & \multicolumn{3}{|c|}{ UN } \\
\hline & & & 10 & 20 & 30 & 10 & 20 & 30 & 10 & 20 & 30 \\
\hline \multirow{16}{*}{$\begin{array}{l}\forall \\
\text { II } \\
2\end{array}$} & \multirow{8}{*}{$\mathbf{C}_{1}$ : } & $T^{2}$ & 178 & 323 & 487 & 226 & 470 & 669 & 183 & 366 & 585 \\
\hline & & $S R I$ & 172 & 325 & 478 & 220 & 450 & 659 & 181 & 361 & 562 \\
\hline & & SSI & 166 & 283 & 409 & 216 & 400 & 585 & 176 & 323 & 510 \\
\hline & & $S R$ & 172 & 326 & 483 & 218 & 450 & 649 & 186 & 359 & 561 \\
\hline & & SS & 157 & 283 & 416 & 198 & 402 & 582 & 168 & 313 & 490 \\
\hline & & $M R$ & 154 & 311 & 460 & 210 & 422 & 631 & 170 & 323 & 528 \\
\hline & & MS & 113 & 174 & 288 & 131 & 282 & 402 & 121 & 187 & 294 \\
\hline & & & & & & & & & & & \\
\hline & \multirow{4}{*}{$\mathbf{C}_{2}$} & $S R$ & 169 & 332 & 481 & 218 & 455 & 658 & 183 & 367 & 567 \\
\hline & & SS & 170 & 292 & 419 & 198 & 412 & 598 & 175 & 329 & 500 \\
\hline & & $M R$ & 153 & 308 & 471 & 210 & 439 & 650 & 171 & 357 & 541 \\
\hline & & $M S$ & 100 & 185 & 307 & 131 & 276 & 460 & 110 & 226 & 314 \\
\hline & \multirow{4}{*}{$\mathbf{C}_{3}$ : } & $S R$ & 170 & 326 & 473 & 216 & 450 & 656 & 180 & 367 & 554 \\
\hline & & $S S$ & 172 & 272 & 409 & 185 & 409 & 578 & 167 & 315 & 502 \\
\hline & & $M R$ & 162 & 306 & 458 & 188 & 435 & 617 & 175 & 365 & 551 \\
\hline & & $M S$ & 117 & 194 & 291 & 138 & 267 & 372 & 128 & 222 & 349 \\
\hline \multirow{16}{*}{$\begin{array}{c}\infty \\
\text { II } \\
2\end{array}$} & \multirow{7}{*}{$\mathbf{C}_{1}:$} & $T^{2}$ & 175 & 421 & 655 & 154 & 388 & 613 & 154 & 398 & 626 \\
\hline & & $S R I$ & 90 & 382 & 619 & 82 & 354 & 560 & 78 & 355 & 594 \\
\hline & & $S S I$ & 86 & 359 & 577 & 76 & 338 & 535 & 85 & 344 & 567 \\
\hline & & $S R$ & 92 & 386 & 618 & 85 & 353 & 564 & 83 & 360 & 605 \\
\hline & & SS & 101 & 378 & 592 & 95 & 344 & 522 & 93 & 353 & 578 \\
\hline & & $M R$ & 72 & 357 & 592 & 62 & 333 & 558 & 70 & 310 & 575 \\
\hline & & $M S$ & 55 & 191 & 332 & 47 & 193 & 333 & 46 & 186 & 351 \\
\hline & \multirow{5}{*}{$\mathbf{C}_{2}$} & & & & & & & & & & \\
\hline & & $S R$ & 80 & 376 & 612 & 84 & 355 & 566 & 81 & 349 & 590 \\
\hline & & $S S$ & 78 & 341 & 579 & 87 & 344 & 535 & 75 & 316 & 553 \\
\hline & & $M R$ & 65 & 316 & 556 & 72 & 326 & 537 & 72 & 286 & 532 \\
\hline & & $M S$ & 47 & 117 & 216 & 45 & 198 & 307 & 54 & 123 & 236 \\
\hline & \multirow{4}{*}{$\mathrm{C}_{3}:$} & $S R$ & 84 & 378 & 611 & 78 & 353 & 567 & 86 & 346 & 587 \\
\hline & & SS & 86 & 351 & 569 & 72 & 336 & 503 & 80 & 321 & 539 \\
\hline & & $M R$ & 70 & 342 & 567 & 59 & 309 & 517 & 67 & 306 & 546 \\
\hline & & $M S$ & 60 & 165 & 302 & 48 & 165 & 247 & 60 & 157 & 274 \\
\hline
\end{tabular}

*Note: The entries within table correspond to empirical powers multiplied by 1000. 


\section{MULTIVARIATE NONPARAMETRIC TESTS IN PROFILE ANALYSIS}

Table 4. The empirical powers of tests under multivariate $t$ distribution.

\begin{tabular}{|c|c|c|c|c|c|c|c|c|c|c|c|}
\hline & \multirow[b]{3}{*}{ Matrix C } & \multirow[b]{3}{*}{ Test } & \multicolumn{9}{|c|}{ Correlation structure } \\
\hline & & & \multicolumn{3}{|c|}{ CS } & \multicolumn{3}{|c|}{ AR1 } & \multicolumn{3}{|c|}{ UN } \\
\hline & & & 10 & 20 & 30 & 10 & 20 & 30 & 10 & 20 & 30 \\
\hline \multirow{16}{*}{$\begin{array}{l}+ \\
\text { II } \\
2\end{array}$} & \multirow{8}{*}{$\mathbf{C}_{1}$ : } & $T^{2}$ & 261 & 480 & 653 & 344 & 607 & 779 & 273 & 527 & 691 \\
\hline & & $S R I$ & 294 & 623 & 822 & 397 & 736 & 919 & 321 & 668 & 872 \\
\hline & & SSI & 309 & 654 & 856 & 427 & 782 & 946 & 336 & 700 & 900 \\
\hline & & $S R$ & 293 & 620 & 823 & 395 & 728 & 920 & 314 & 667 & 871 \\
\hline & & SS & 317 & 648 & 840 & 414 & 771 & 940 & 328 & 689 & 889 \\
\hline & & $M R$ & 264 & 587 & 788 & 370 & 713 & 915 & 263 & 601 & 816 \\
\hline & & $M S$ & 180 & 410 & 582 & 287 & 511 & 721 & 176 & 391 & 602 \\
\hline & & & & & & & & & & & \\
\hline & \multirow{4}{*}{$\mathbf{C}_{2}$} & $S R$ & 293 & 616 & 818 & 402 & 735 & 918 & 322 & 674 & 872 \\
\hline & & $S S$ & 315 & 653 & 846 & 424 & 782 & 946 & 342 & 691 & 891 \\
\hline & & $M R$ & 276 & 591 & 802 & 380 & 720 & 922 & 286 & 643 & 846 \\
\hline & & $M S$ & 180 & 401 & 636 & 263 & 537 & 795 & 179 & 387 & 661 \\
\hline & \multirow{4}{*}{$\mathbf{C}_{3}$ : } & $S R$ & 287 & 635 & 826 & 397 & 733 & 918 & 326 & 670 & 863 \\
\hline & & SS & 303 & 658 & 841 & 401 & 756 & 939 & 343 & 695 & 893 \\
\hline & & $M R$ & 278 & 591 & 793 & 359 & 711 & 903 & 294 & 630 & 847 \\
\hline & & $M S$ & 219 & 432 & 642 & 263 & 544 & 759 & 212 & 495 & 723 \\
\hline \multirow{16}{*}{$\begin{array}{l}\infty \\
\text { II } \\
2\end{array}$} & \multirow{7}{*}{$\mathbf{C}_{1}$} & $T^{2}$ & 317 & 632 & 822 & 267 & 590 & 773 & 296 & 607 & 799 \\
\hline & & $S R I$ & 194 & 709 & 917 & 169 & 661 & 893 & 184 & 669 & 902 \\
\hline & & $S S I$ & 196 & 761 & 953 & 170 & 716 & 934 & 180 & 731 & 940 \\
\hline & & $S R$ & 201 & 717 & 920 & 175 & 676 & 900 & 195 & 682 & 911 \\
\hline & & $S S$ & 233 & 776 & 954 & 205 & 729 & 931 & 212 & 743 & 945 \\
\hline & & $M R$ & 176 & 681 & 893 & 145 & 638 & 868 & 166 & 653 & 878 \\
\hline & & $M S$ & 108 & 444 & 718 & 112 & 405 & 695 & 113 & 439 & 722 \\
\hline & \multirow{5}{*}{$\mathbf{C}_{2}$} & & & & & & & & & & \\
\hline & & $S R$ & 200 & 709 & 916 & $1 / 2$ & $6 / 8$ & 899 & 183 & 669 & 906 \\
\hline & & SS & 190 & 760 & 947 & 195 & 730 & 931 & 172 & 719 & 939 \\
\hline & & $M R$ & 151 & 607 & 870 & 149 & 635 & 866 & 149 & 578 & 840 \\
\hline & & $M S$ & 101 & 268 & 504 & 099 & 378 & 632 & 102 & 257 & 496 \\
\hline & \multirow{4}{*}{$\mathrm{C}_{3}$ : } & $S R$ & 200 & 711 & 917 & 161 & 667 & 901 & 178 & 672 & 902 \\
\hline & & SS & 218 & 765 & 946 & 176 & 693 & 923 & 197 & 718 & 930 \\
\hline & & $M R$ & 171 & 634 & 890 & 135 & 601 & 836 & 154 & 608 & 859 \\
\hline & & $M S$ & 119 & 414 & 694 & 100 & 349 & 606 & 126 & 379 & 645 \\
\hline
\end{tabular}

*Note: The entries within table correspond to empirical powers multiplied by 1000.

Except for the test based on marginal sign, the performances of other non invariant tests were relatively robust with respect to different choices of transformation matrix $\mathbf{C}$ to test parallelism. There was not a unique choice for $\mathbf{C}$ which corresponded to the best performance of the tests. Figure 3 illustrates the degree of stability in power values for the 4 non-invariant tests for the three transformation matrices $\mathbf{C}_{1}-\mathbf{C}_{3}$ when $n=30$. 


\section{VOSSOUGHI ET AL.}

$\boldsymbol{\Sigma}$
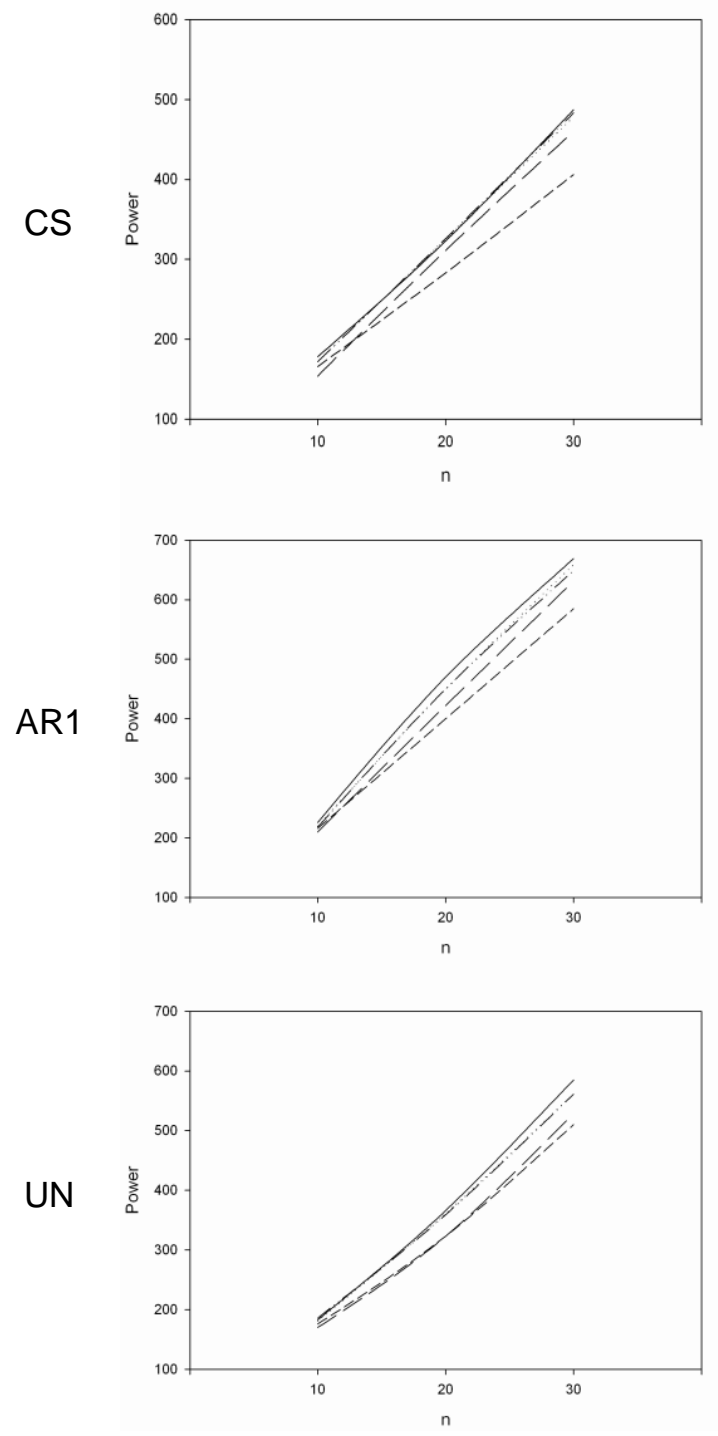

$T^{2}$

(a): $p=4$
SRI $\quad----S S I$
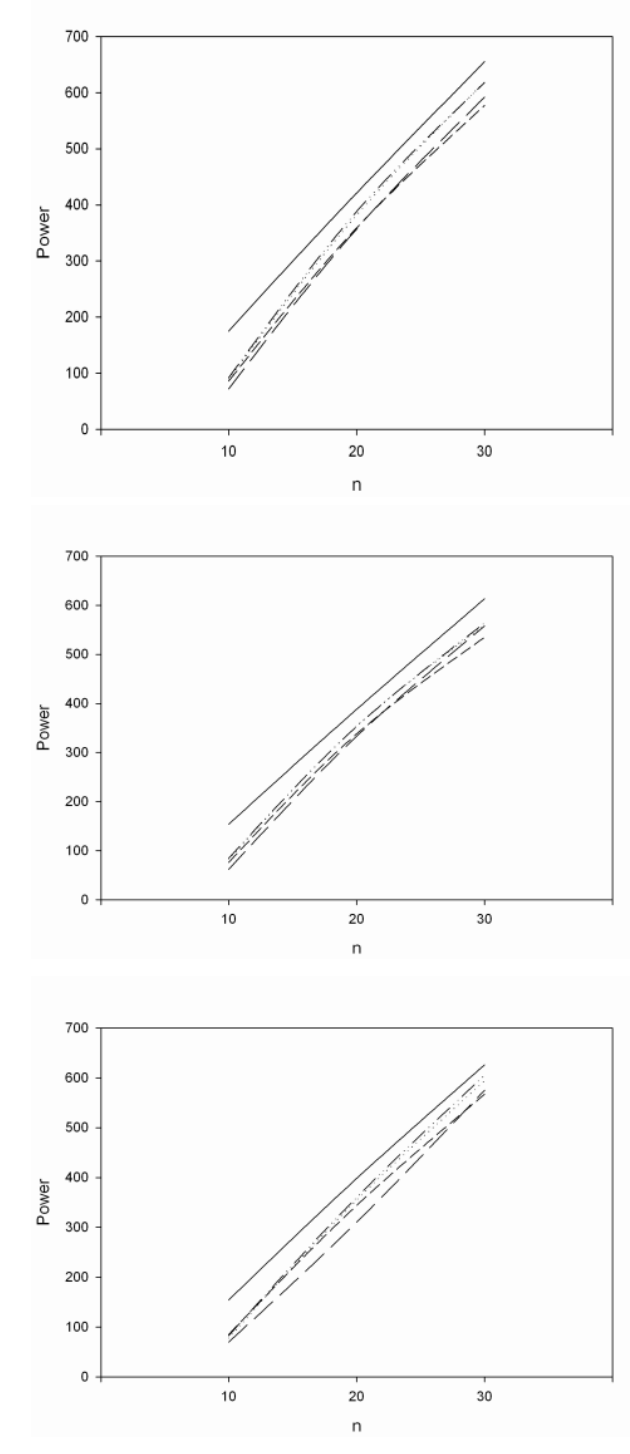

(b): $p=8$

$\mathrm{MR}-\mathrm{MS}$

*Note: For purpose of better illustration, the powers of non-invariant tests are displayed only for the matrix $\mathbf{C}_{2}$.

Figure 1. The empirical powers of tests under multivariate normal distribution. 


\section{MULTIVARIATE NONPARAMETRIC TESTS IN PROFILE ANALYSIS}

$\boldsymbol{\Sigma}$

$$
\text { (a): } p=4
$$
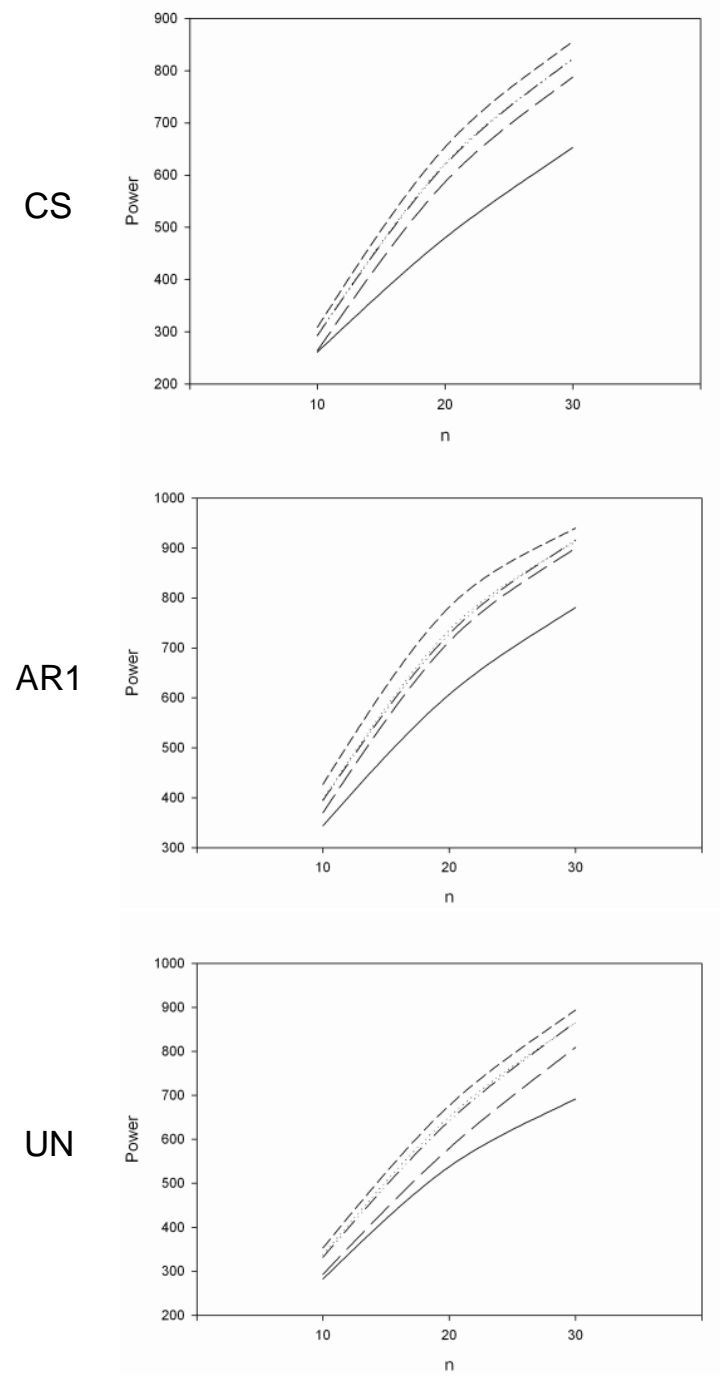

$T^{2}$

SRI

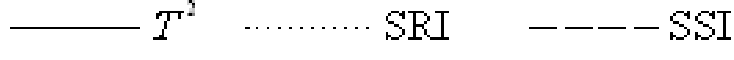

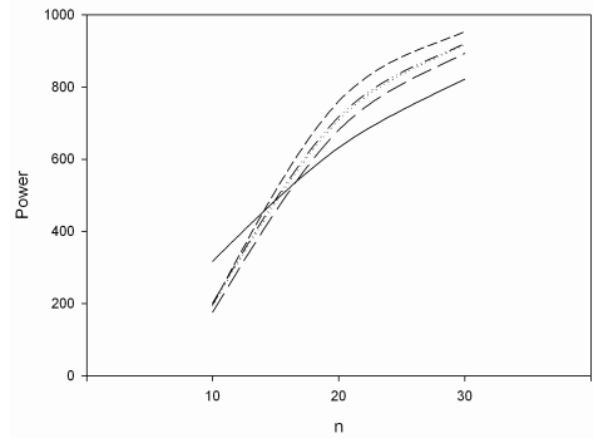
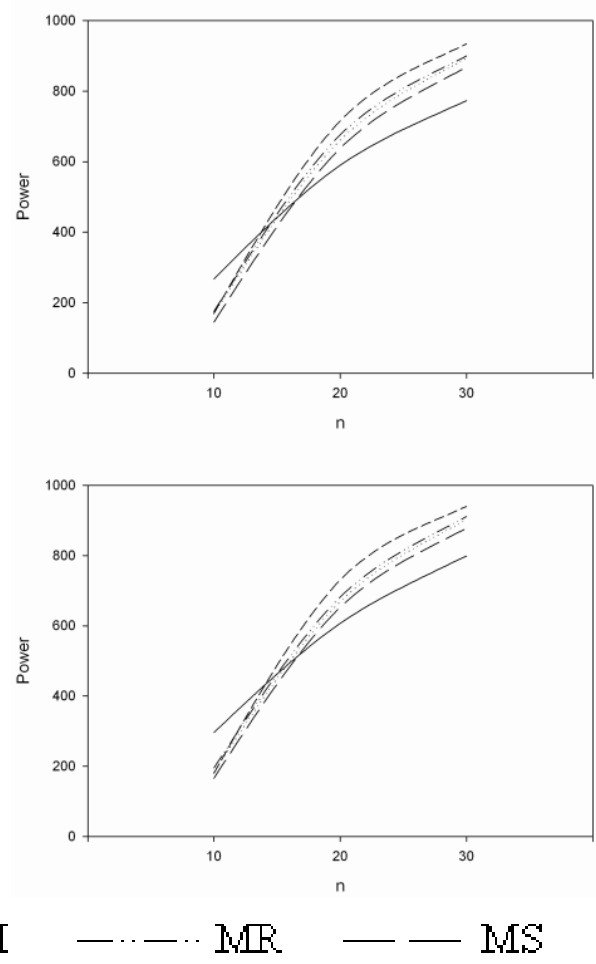

*Note: For purpose of better illustration, the powers of non-invariant tests are displayed only for the matrix $\mathbf{C}_{2}$.

Figure 2. The empirical powers of tests under multivariate $t(3)$ distribution. 
$\boldsymbol{\Sigma}$

(a)
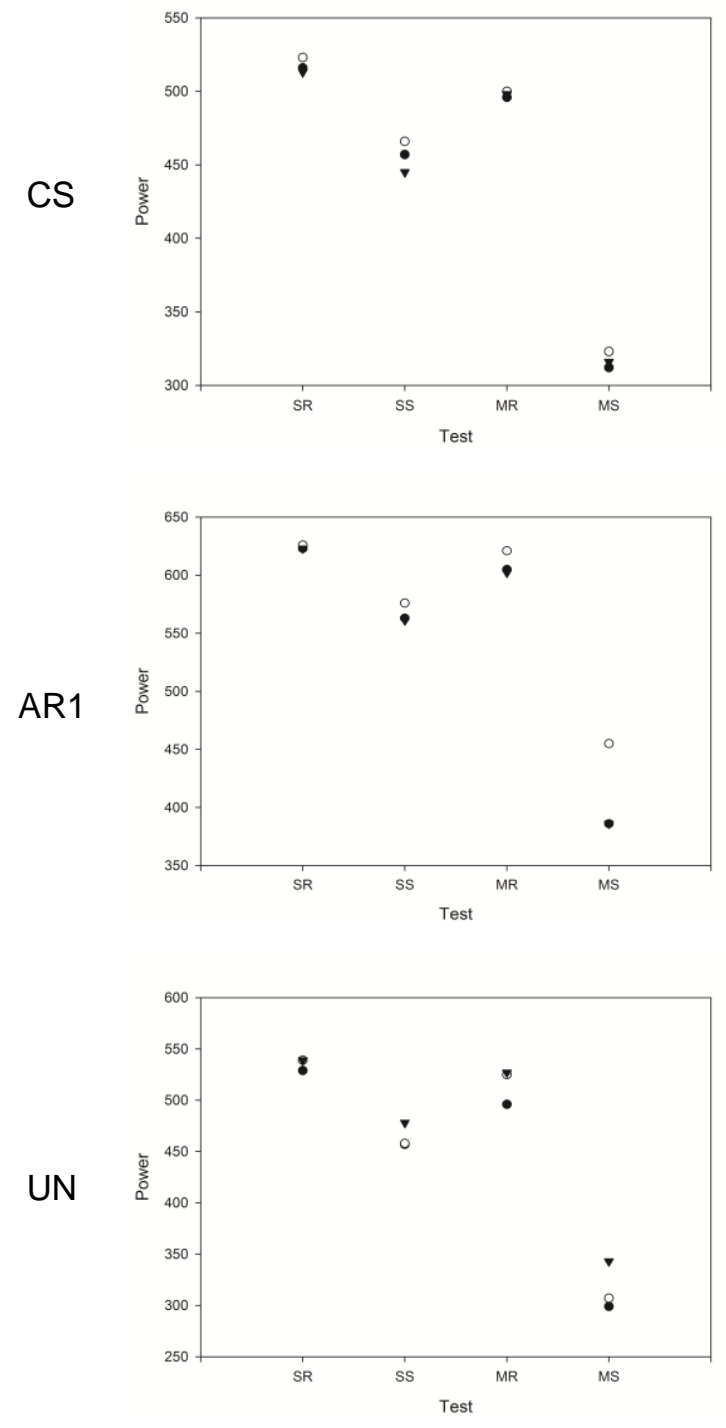

symbol key: $\bullet: \mathbf{C}_{\mathbf{1}}, \circ$ : $\mathbf{C}_{\mathbf{2}}$, and $\boldsymbol{\nabla}: \mathbf{C}_{\mathbf{3}}$

(b)
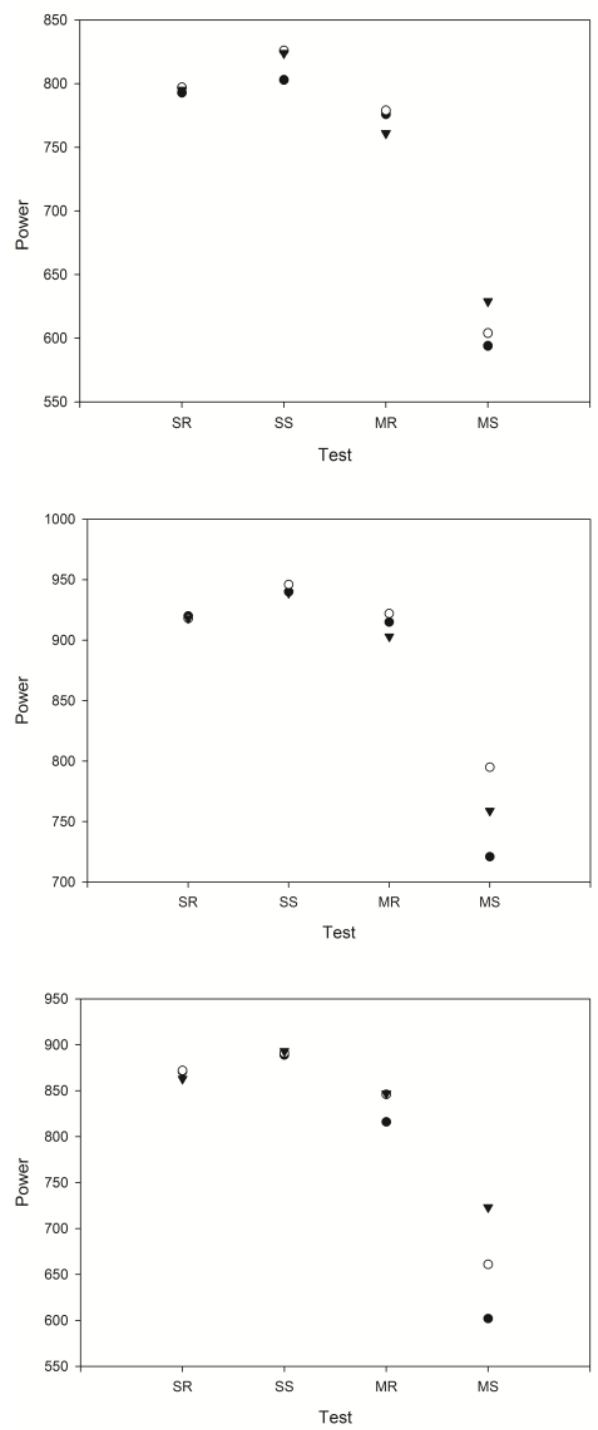

Figure 3. The empirical powers of non-invariant tests for $n=30$ for various transformation matrices under multivariate normal (a) and $t$ (b) distributions 


\section{MULTIVARIATE NONPARAMETRIC TESTS IN PROFILE ANALYSIS}

\section{Conclusion}

The results of the study revealed that the tests based on spatial and marginal ranks could serve as efficient tools for profile analysis since they performed notably better than Hotelling's $T^{2}$ for the heavy tailed distribution and were as efficient as it under normality. Similar results reported in simulation studies by Nordhausen et al. (2006) and Möttönen et al. (1998) only in the context of two sample comparison of locations for normal and $t$ distributions. Interestingly, even for moderate tailed $t$ distributions, the tests based on ranks were superior to Hotelling's $T^{2}$ in both studies. Um and Randles (1998) also reported that the multi sample extensions of multivariate rank tests proposed by Randles and Peters (1990) were more efficient than Lawly-Hotelling's U for light-tailed and heavytailed distributions. However, the results revealed that when there was sufficient evidence to conclude that the underlying distribution was heavy tailed, the tests based on spatial signs were the best choices to profile analysis. Similarly, this aspect was reported in the study by Nordhausen et al. (2006) and for a different sign test by Um and Randles (1998). It should also be noted that above studies conducted in areas not involving repeated measurements and various correlation structures for errors. The simulations also illustrated that when the number of replication was large (here $p=8$ ) the mentioned nonparametric tests outperformed Hotelling's $T^{2}$ only for larger sample sizes $(n \geq 10)$. The panel (b) of Figure 2 illustrated this issue for which Hotelling's $T^{2}$ performed slightly better than any nonparametric counter parts for $p=8$ even if the underlying distribution was heavy-tailed. The effect of sample size relative to the number of measurements has been not reported yet and hence further research in this area is necessary.

In the context of two sample comparison (as our study), Hotelling's $T^{2}$ and all the MANOVA tests (Wilks' $\boldsymbol{\Lambda}$, Pilla's V, Lawley-Hotelling's $\mathbf{U}$ and Roy's $\boldsymbol{\theta}$ ) are functions of each other and give equivalent results; see Rencher (1998). The power of the MANOVA tests has been compared by several authors. However, they are asymptotically equivalent for sufficient sample sizes (Olson, 1974). Therefore it is implied the nonparametric alternatives can be confidently applied in place of MANOVA tests in profile analysis regardless of the nature of underlying distribution. Park et al. (2001) investigated the performance of profile analysis using Hotelling's $T^{2}$ and mixed model approach to test group and interaction effects. Also, Vossoughi et al. (2012) compared the performance of profile analysis, linear mixed model and summary measure approach in repeated measurements generated from a linear mixed model setting. Similarly, both studies showed that the profile analysis preserved the nominal significance level 


\section{VOSSOUGHI ET AL.}

and performed relatively robust to the underlying correlation structure but provided less power values than the competitors, in general.

Marcucci (1986) demonstrated that profile analysis using Hotelling's $T^{2}$ and exclusively univariate split-plot analysis with d.f. adjustments gave type I error rates closest to the nominal level, but not one of which was most powerful along various correlation structures and patterns of means. The interested reader is also referred to Schwertman et al. (1985), Boik (1991) and Davidson (1972) for further assessment on this issue.

Thought not reported here, we conducted additional simulations for a variety type of the location trend over occasions such as linear trend as $\boldsymbol{\theta}_{2}=(0.25,0.5,0.75,1)^{\prime}$. The larger number of measurements $p=8$ and sample size $n=50$ in each group were also considered. However, the similar results were yielded and hence not further included in the study.

In conclusion, the findings implied that the use of some nonparametric multivariate tests in place of the parametric counterparts can considerably improve the result of profile analysis for heavy-tailed distributions. Accordingly, the tests based on spatial and marginal ranks are severe competitors for parametric tests in profile analysis since they performed as well as Hotelling's $T^{2}$ under multivariate normal distribution and dominated it under heavy-tailed distribution. Moreover, the simulation results revealed that the tests based on spatial signs under heavy tailed distributions, were more efficient than the MANOVA tests for the analysis of repeated measurements.

\section{References}

Azzalini, A., \& Bowman, A. (1991). Nonparametric regression methods for repeated measurements. In G. Roussas (Ed.), Nonparametric functional estimation and related topics (pp. 377-387). Netherlands: Springer.

Bhapkar, V. P. (1984). Univariate and multivariate multisample location and scale tests. In P. R. Krishnaiah \& P. K. Sen (Eds.), Nonparametric methods (pp. 31-62). Oxford: Elsevier.

Bhapkar, V. P., \& Patterson, K.W. (1978). A Monte Carlo study of some multivariate nonparametric statistics for profile analysis of several samples. Journal of Statistical Computation and Simulation, 6(3/4), 223-237. doi: 10.1080/00949657808810191 


\section{MULTIVARIATE NONPARAMETRIC TESTS IN PROFILE ANALYSIS}

Boik, R. J. (1991). Scheffés mixed model for multivariate repeated measures: A relative efficiency evaluation. Communications in Statistics - Theory and Methods, 20(4), 1233-1255. doi: 10.1080/03610929108830562

Davidson, M. L. (1972). Univariate versus multivariate tests in repeatedmeasures experiments. Psychological Bulletin, 77(6), 446-452. doi: $10.1037 / \mathrm{h} 0032674$

Davis, A. W. (1980). On the effects of moderate multivariate nonnormality on Wilks's likelihood ratio criterion. Biometrika, 67(2), 419-427.

doi: 10.1093/biomet/67.2.419

Davis, A. W. (1982). On the effects of moderate multivariate nonnormality on Roy's largest root test. Journal of the American Statistical Association, 77(380), 896-900. doi: 10.1080/01621459.1982.10477904

Davis, C. S. (2002). Statistical methods for the analysis of repeated measurements. New York, NY: Springer-Verlag.

Everitt, B. S. (1979). A Monte Carlo investigation of the robustness of Hotelling's one-and two-sample $T^{2}$ tests. Journal of the American Statistical Association, 74(365), 48-51. doi: 10.1080/01621459.1979.10481606

Hettmansperger, T. P., Möttönen, J. \& Oja, H. (1998). Affine invariant multivariate rank tests for several samples. Statistica Sinica, 8(3), 785-800.

Hettmansperger, T. P., \& Oja, H. (1994). Affine invariant multivariate multisample sign tests. Journal of the Royal Statistical Society. Series B (Methodological), 56(1), 235-249.

Laird, N. M., \& Ware, J. H. (1982). Random-effects models for longitudinal data. Biometrics, 38(4), 963-974. doi: 10.2307/2529876

Littell, R. C., Pendergast, J., \& Natarajan, R. (2000). Tutorial in biostatistics: Modelling covariance structure in the analysis of repeated measures data. Statistics in Medicine, 19, 1793-1819.

Marcucci, M. (1986). A comparison of the power of some tests for repeated measurements. Journal of Statistical Computation and Simulation, 26(1/2), 37-53. doi: 10.1080/00949658608810948

Mendoza, J. L., Toothaker, L. E., \& Nicewander, W. A. (1974). A Monte Carlo comparison of the univariate and multivariate methods for the groups by trials repeated measures design. Multivariate Behavioral Research, 9(2), 165-177. doi: 10.1207/s15327906mbr0902_3 


\section{VOSSOUGHI ET AL.}

Möttönen, J., Hettmansperger, T. P., Oja, H., \& Tienari, J. (1998). On the efficiency of affine invariant multivariate rank tests. Journal of Multivariate Analysis, 66(1), 118-132. doi: 10.1006/jmva.1998.1740

Möttönen, J., \& Oja, H. (1995). Multivariate spatial sign and rank methods. Journal of Nonparametric Statistics, 5(2), 201-213. doi: 10.1080/10485259508832643

Möttönen, J., Oja, H., \& Tienari, J. (1997). On the efficiency of multivariate spatial sign and rank tests. The Annals of Statistics, 25(2), 542-552.

doi: $10.1214 /$ aos/1031833663

Nordhausen, K., Oja, H., \& Tyler, D. E. (2006). On the efficiency of invariant multivariate sign and rank tests. In E. P. Liski, J. Isotalo, J. Niemelä, S. Puntanen \& G. P. H. Styan (Eds.), Festschrift for Tarmo Pukkila on his 60th birthday (pp.217-231). Tampere, Finland: Dept. of Mathematics, Statistics and Philosophy, University of Tampere.

Oja, H. (1999). Affine invariant multivariate sign and rank tests and corresponding estimates: A review. Scandinavian Journal of Statistics, 26(3), 319-343. doi: 10.1111/1467-9469.00152

Oja, H. (2010). Multivariate nonparametric methods with R: An approach based on spatial signs and ranks. New York, NY: Springer.

Oja, H., \& Randles, R. H. (2004). Multivariate nonparametric tests. Statistical Science, 19(4), 598-605. doi: 10.1214/088342304000000558

Olson, C. L. (1974). Comparative robustness of six tests in multivariate analysis of variance. Journal of the American Statistical Association, 69(348), 894-908.

Park, T., Park, J.-K., \& Davis, C. S. (2001). Effects of covariance model assumptions on hypothesis tests for repeated measurements: Analysis of ovarian hormone data and pituitary-pteryomaxillary distance data. Statistics in Medicine, 20(16), 2441-2453. doi: 10.1002/sim.859

Puri, M. L., \& Sen, P. K. (1971). Nonparametric methods in multivariate analysis. New York, NY: Wiley.

R Development Core Team. (2013). R: A language and environment for statistical computing. Reference index (Version 3.0.1). Vienna, Austria: R Foundation for Statistical Computing.

Randles, R. H., \& Peters, D. (1990). Multivariate rank tests for the twosample location problem. Communications in Statistics - Theory and Methods, 19(11), 4225-4238. doi: 10.1080/03610929008830439 


\section{MULTIVARIATE NONPARAMETRIC TESTS IN PROFILE ANALYSIS}

Rencher, A. C. (1995). Methods of multivariate analysis. New York, NY: Wiley.

Rencher, A. C. (1998). Multivariate statistical inference and applications. New York, NY: Wiley.

Schwertman, N. C., Flynn, W., Stein, S., \& Schenk, K. L. (1985). A Monte Carlo study of alternative procedures for testing the hypothesis of parallelism for complete and incomplete growth curve data. Journal of Statistical Computation and Simulation, 21(1), 1-37. doi: 10.1080/00949658508810794

Schwertman, N. C., Fridshal, D., \& Magrey, J. M. (1981). On the analysis of incomplete growth curve data, a Monte Carlo study of two nonparametric procedures. Communications in Statistics-Simulation and Computation, 10(1), 51-66. doi: 10.1080/03610918108812192

Sen, P. K. (1984). Nonparametric procedures for some miscellaneous problems. In P. R. Krishnaiah \& P. K. Sen (Eds.), Nonparametric methods (pp. 699-739). Oxford: Elsevier.

Singer, J. M., Poleto, F. Z., \& Rosa, P. (2004). Parametric and nonparametric analyses of repeated ordinal categorical data. Biometrical Journal, 46(4), 460-473. doi: 10.1002/bimj.200310045

Somorčík, J. (2006). Tests using spatial median. Austrian Journal of Statistics, 35(2/3), 331-338. doi: 10.17713/ajs.v35i2\&3.380

Um, Y., \& Randles, R. H. (1998). Nonparametric tests for the multivariate multi-sample location problem. Statistica Sinica, 8(3), 801-812.

Vossoughi, M., Ayatollahi, S. M. T., Towhidi, M., \& Ketabchi, F. (2012). On summary measure analysis of linear trend repeated measures data: performance comparison with two competing methods. BMC Medical Research Methodology, 12(33), 1-12. doi: 10.1186/1471-2288-12-33

Ware, J. H. (1985). Linear models for the analysis of longitudinal studies. American Statistician, 39(2), 95-101.

Wernecke, K.-D., \& Kalb, G. (1999). On nonparametric repeated measures analysis of variance. In W. Gaul \& H. Locarek-Junge (Eds.), Classification in the information age (pp. 596-604). Berlin: Springer.

Wernecke, K.-D., \& Kaufmann, J. (2000). A new approach on nonparametric repeated measures analysis of variance. In R. Decker \& W. Gaul (Eds.), Classification and information processing at the turn of the millennium (pp. 430-437). Berlin: Springer. 